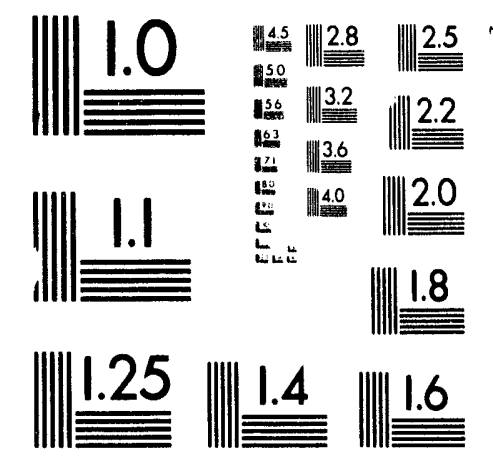



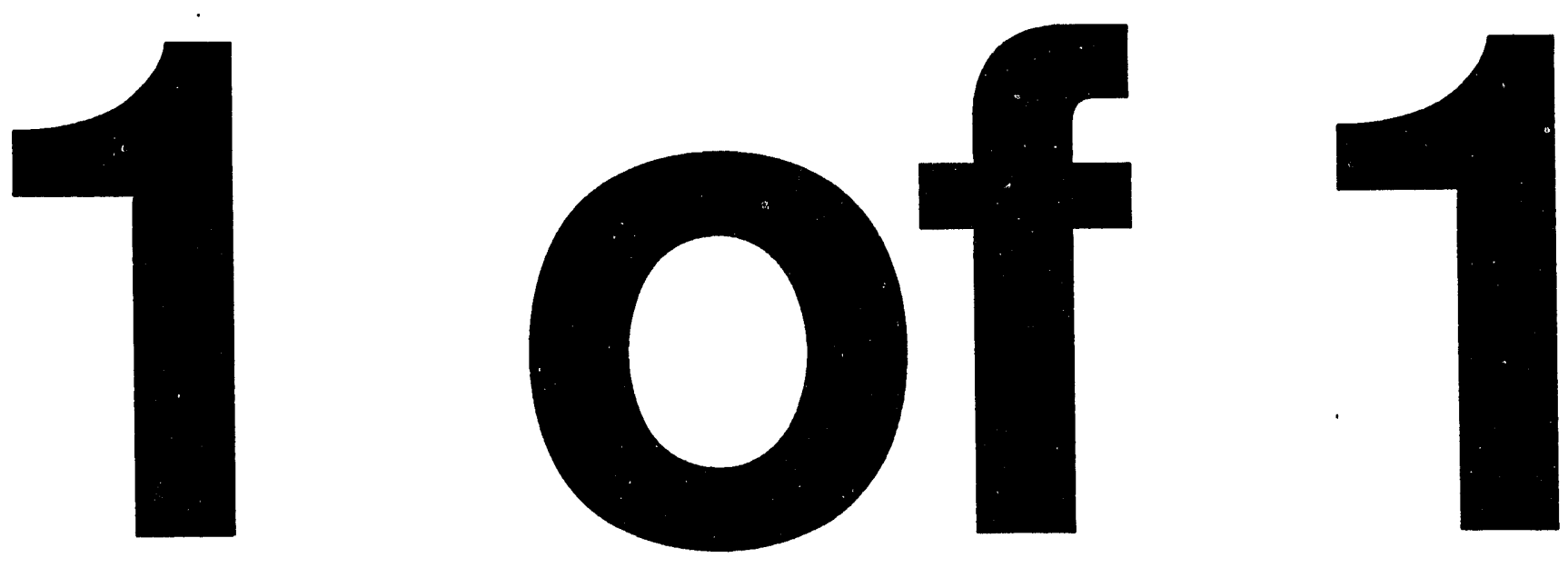


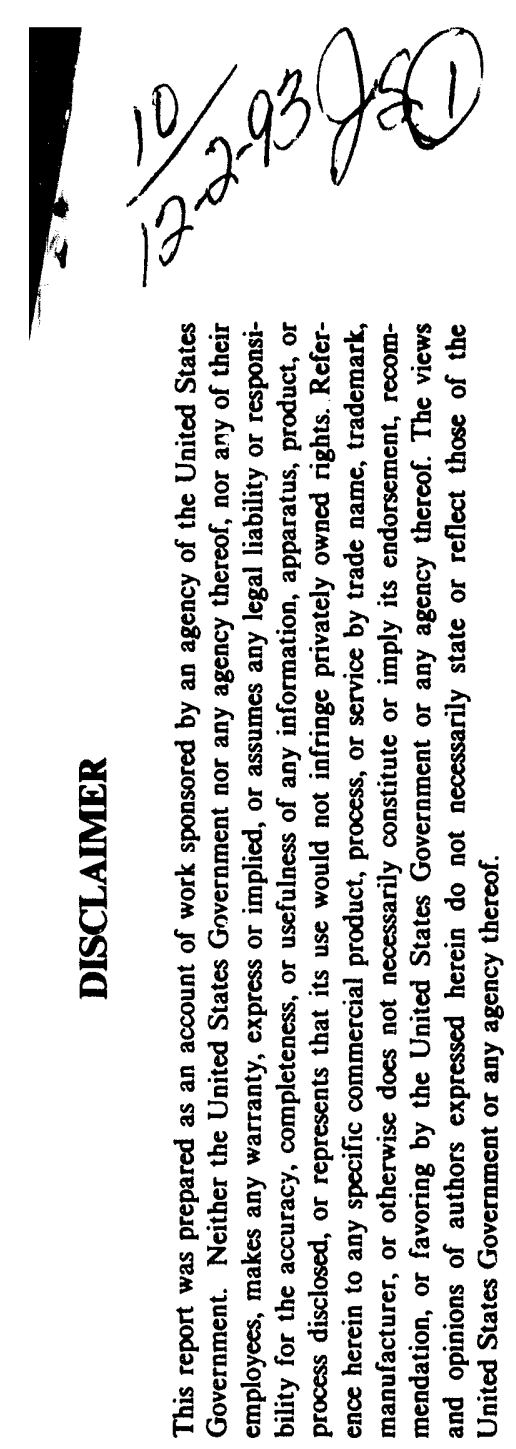

SLAC-PUB-4252

March 1987

(N)

\title{
BEAMSTRAHLUNG MONITORS AT SLC *
}

\author{
R. C. FIELD \\ Stanford Linear Accelerator Center \\ Stanford University, Stanford, California, 94305
}

Some properties of beamstrahlung are discussed, and plans to observe the effect at SLC by the use of Cherenkov counters are illustrated.

Invited talk presented at the International Conference on Advances in Experimental Methods for Colliding Beam Physics, Stanford, California, March 9-13, 1987

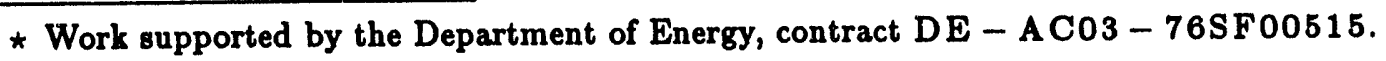


Monitoring of the SLC targeting accuracy requires methods quite different from those used on present-day storage rings. Interaction rates will be low. Even for Bhabha scatters into the $15-25$ mrad region, which is the limit of accessibility, rates of less than 10 per hour are expected at the start, with luminosities, $L$, below $10^{28} \mathrm{~cm}^{-2} \mathrm{sec}^{-1}$. As an alternative, the most energetic process during beam-beam collisions has been examined. This is the deflection of trajectories in the collective field of the opposing bunch.

Under SLC conditions, with bunches 2-20 microns wide, $\sim 2 \mathrm{~mm}$ long and containing 1-5 $\times 10^{10}$ particles, the peripheral fields can approach $10^{6}$ gauss. Deflection of the average trajectory directions caused by this should be measurable when beams are mis-targeted by distances up to a few times the width of the bunches [1]. Obviously there must be synchrotron radiation under these conditions. This is "beamstrahlung", but although it has been the subject of theoretical work [2], it has yet to be observed.

The classical rules of synchrotron radiation apply [3] - quantum corrections are not needed under SLC conditions - except that, since the fields are not uniform, one must integrate the spatial overlap of the electron density in a bunch with the square of the field of the target bunch, and integrate along the bunch length as well.

In the case of Gaussian shaped bunches analytic calculations have been made (for example, Bassetti et al., ref. [2]). The energy radiated by beam 1 in head-on collision with beam 2 is

$$
W=\frac{0.22 r^{3} \gamma^{2} m c^{2} N_{1} N_{2}^{2}}{\sigma_{x} \sigma_{y} \sigma_{z}} \propto \frac{L N_{2} \gamma^{2}}{\sigma_{z}},
$$

where $r$ is the classical radius and $m$ the mass of the electron, $\gamma$ is the Lorentz 
factor, $L$ the luminosity per collision, $N_{1}$ and $N_{2}$ the populations of the two bunches, $\sigma_{x}, \sigma_{y}$ the transverse rms bunch widths, and $\sigma_{z}$ the bunch rms length. Although there is a strong dependence on luminosity, other factors, notably $N_{2}$, complicate the relation.

A further property of interest is that the characteristic cone angle of the beamstrahlung radiation is $\left\langle q^{-1}\right.$ relative to the emitting electron trajectory. Since this is below $10 \mu \mathrm{rad}$ at SLC compared with $>200 \mu \mathrm{rad}$ for the electron beam divergence at the interaction point, the transverse distribution of beamstrahlung at the plane of a detector describes the full range of electron trajectories during the collision, including deflection.

The radiation may only be detected after the electron beam has been deflected outside the bounds of the beamstrahlung cone. This deflection, in turn, produces synchrotron radiation which still overlaps the beamstrahlung envelope. Since it corresponds to the lower field of an electromagnet, its characteristic energy is low, even though the magnetic length ensures that intensities are high. At SLC, the only available detector position, $41 \mathrm{~m}$ from the interaction point, will be subject to 10 watts of synchrotron radiation with a critical energy of $2.3 \mathrm{MeV}$.

The mean critical energy of beamstrahlung is expected to be an order of magnitude larger, and this yields the means of separating the signal from the synchrotron radiation background.

A few percent of the gamma rays can be converted into $e^{+} e^{-}$pairs without the converter thickness causing severe loss by multiply scattering the charged particles. The option of using a spectrometer to select beamstrahlung positrons cleanly has been rejected because of inadequate room. The favored option is to use Cherenkov counters with a threshold in the range of $25 \mathrm{MeV}$. 
Because of the threshold, and the need for flexibility in the beam parameters, numerical simulations are necessary to establish the properties of the detected signal with respect to collision conditions.

The group working on SLC beamstrahlung detector development includes G. Bonvicini, R. Frey, E. Gero, W. Koska, A. Minten and the author. The simulations it has carried out are illustrated in fig. 1, where the high energy fanma ray fluxes are plotted against beam-beam targeting errors, expressed in terms of the beam rms widths, for various beam conditions. The $120 \mathrm{~Hz}$ luminosities indicated correspond to head-on collisions, and include an enhancement factor [4] caused by the disruptive collapse of the beam width during collisions with $L Z 10^{30}$. The almost universal shape of the curves at higher luminosity is noteworthy, as is the failure of the universality at lower luminosities where the threshold energy exceeds the critical energy of the radiation.

In fig. 2 is illustrated the deflection of the beamstrahlung beam as the charged beams are mis-steered relative to each other. Since measurements of the beamstrahlung deflection to $5 \mu \mathrm{rad}$ are believed to be possible systematically and statistically, the technique should eventually lead to a means of monitoring the collider steering, pulse by pulse.

The synchrotron radiation from the deflecting magnet will generate backgrounds which we have found difficult to evaluate. Compton scattering in the vacuum pipe window, counter body, etc., will generate a sea of quite penetrating photons. On rescattering in the Cherenkov light sensitive elements, they could overwhelm the detectors with ionization. In response, the device has been designed with photomultiplier tubes, and these are placed at the end of an indirect light path, and heavily shielded with lead. 
Another difficulty comes from Compton scattering in the counter gas, where the recoil electrons excite scintillation. The use of nitrogen as the Cherenkov gas would lead to a scintillation background equivalent to the beamstrahlung signal for a luminosity of $10^{29}$. The use of ethylene [5] at 0.3 atmospheres will suppress this problem.

It is believed that other backgrounds, for example, associated with neutron production by the beamstrahlung itzelf, will be tolerable. Damage to the primary mirror by the intense bombardment is possible, but there is no information to help establish $:$ uffect on the signal to noise ratio.

A schematic perspective drawing of the optical layout of a counter is given in fig. 3. The scattering of the pairs produced in the converter plate is cancelled optically by imaging the plate at the photomultiplier tubes. Light is collected at a waist approximately one focal length from the primary mirror. From there it is reflected into a narrow light channel where the vertical spread is compressed between parallel mirrors. The horizontal spread is undisturbed until it reaches the image plane where the photomultiplier tubes allow us to reconstruct the horizontal projection of the beamstrahlung "spot". In fact the horizontal distribution is to be monitored on the south side of the interaction point, while the vertical distribution will be observed on the north side.

Estimates of the photoelectron yield of the device for head-on collisions are shown in fig. 4. The curvature of the lines is a result of the $25 \mathrm{MeV}$ detector threshold. It is evident that the yield is large but has a wide range. In fact the photomultipliers will saturate excessively above $L=10^{29}$, and it will, later, be necessary to mask the faces or find tubes with higher pulse current capabilities. 
The electronics are quite conventional commercial equipment, and are selected to be serviceable as SLC electronics pool components.

The initial task will be to face whatever unforeseen problems arise, and to extract a beamstrahlung signal. Then we may attempt to mold it into a tool for beam collision diagnostics. 


\section{References}

[1] P. Bambade, SLAC-CN-303, June 1985.

[2] M. Bassetti et al., IEEE Trans. Nucl. Sci. NS-30 (1983) 2182; R.J. Noble, SLAC-AAS-Note 3, December 1985. Also references therein.

[3] M. Sands, SLAC-121, November 1970; J.D. Jackson, Classical Electrodynamics, Wiley, 1975.

[4] R. Hollebeek, Nucl. Instrum. Methods 184 (1981) 333.

[5] J. Heintze et al., Nucl. Instrum. Methods 138 (1976) 641. 


\section{Figure Captions}

Fig. 1. Gamma ray yields above $20 \mathrm{MeV}$ plotted against beam-beam missteering in units of rms bunch width. Indicated luminosities, $L$, assume head-on collisions at $120 \mathrm{~Hz}$.

Fig. 2. Deflection of the center of the beamstrahlung versus beam-beam mis-steering, for two different bunch widths.

Fig. 3. Perspective illustration of the optical components of a counter: C, converter plate; P, primary mirror; L, light channel of parallel reflectors with two $90^{\circ}$ reflections; $\mathrm{T}$, photomultiplier tubes.

Fig. 4. Expected yield, in photoelectrons, versus luminosity, for bunches of (A) $5 \times 10^{10}$ and (B) $2.5 \times 10^{10}$ particles per pulse. 

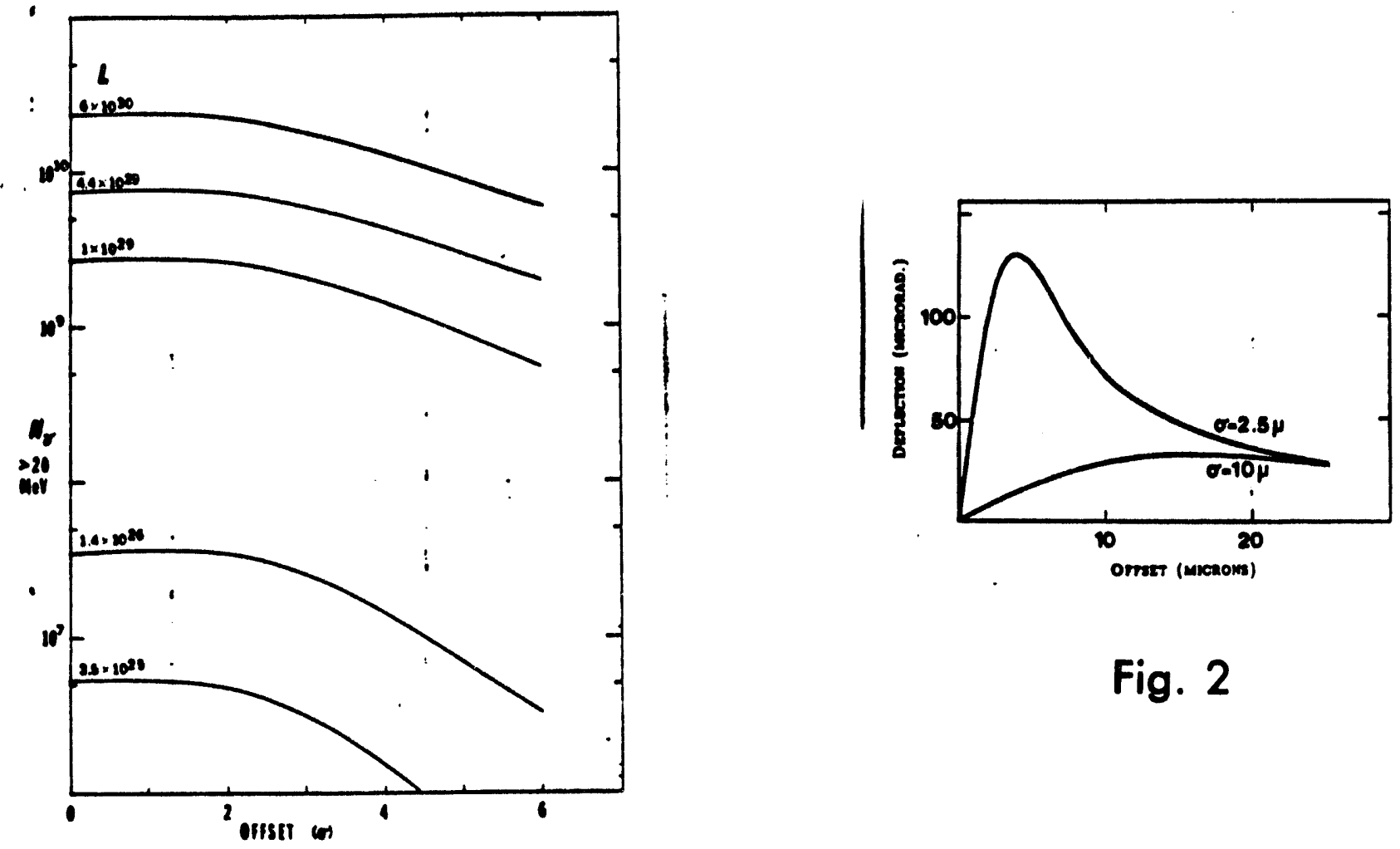

Fig. 1

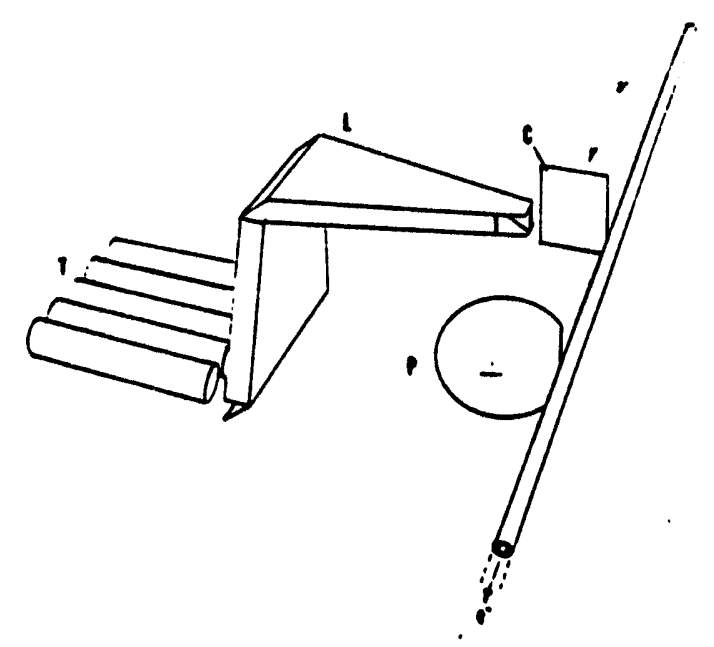

Fig. 3

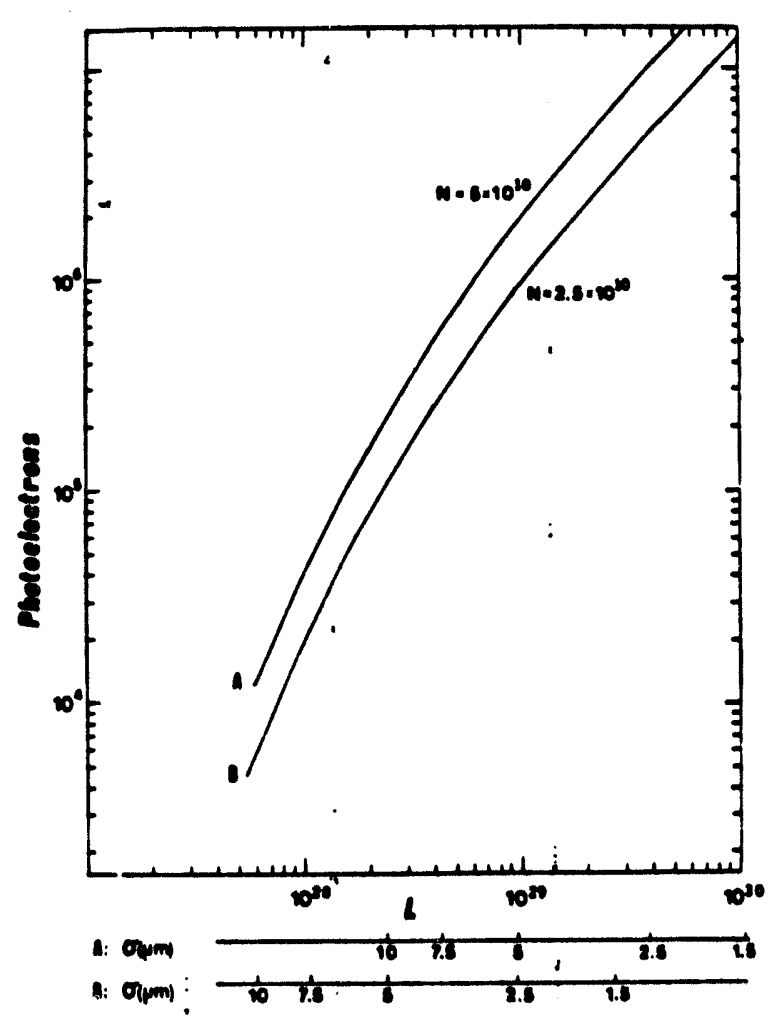

Fig. 4 

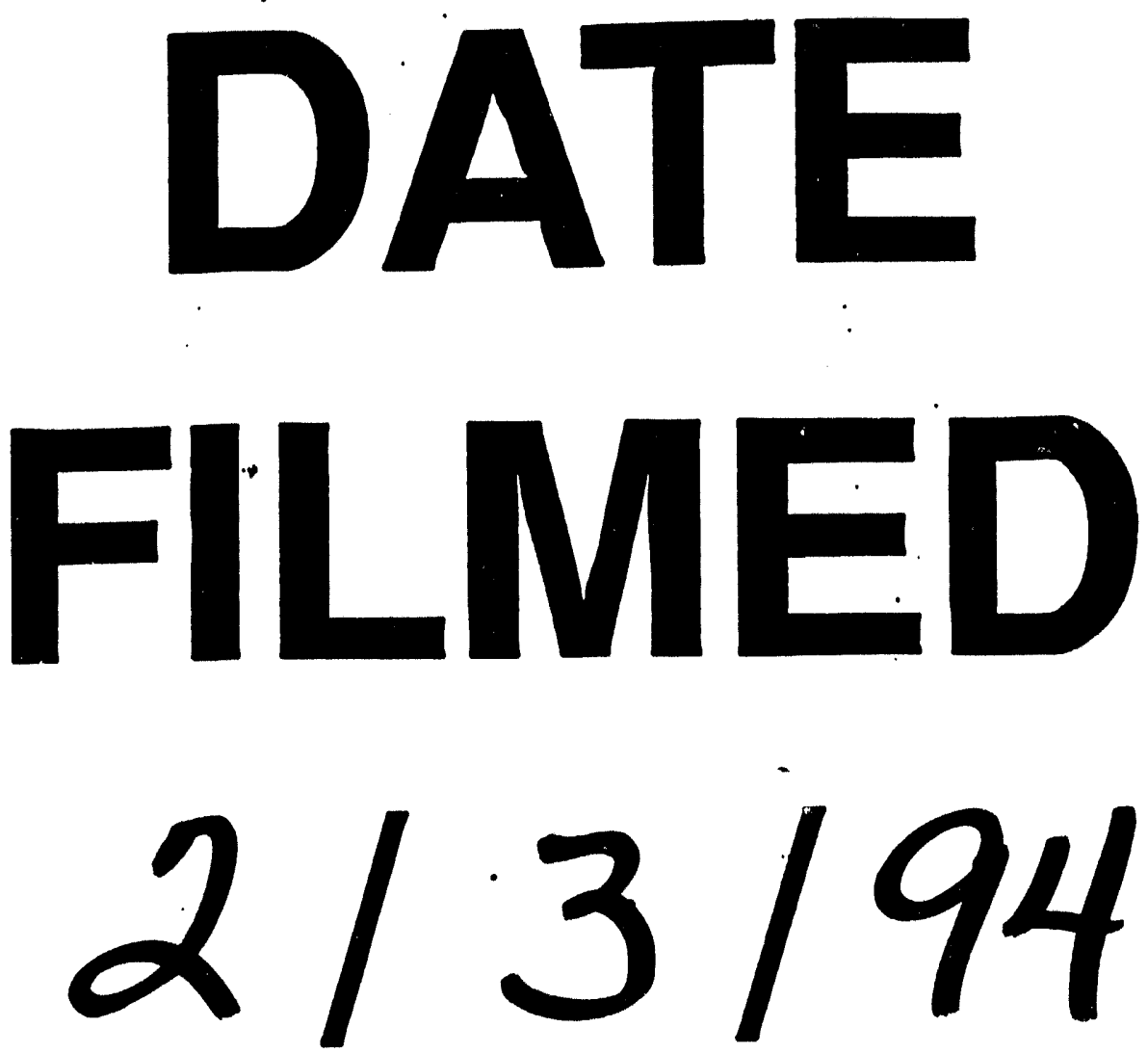

94
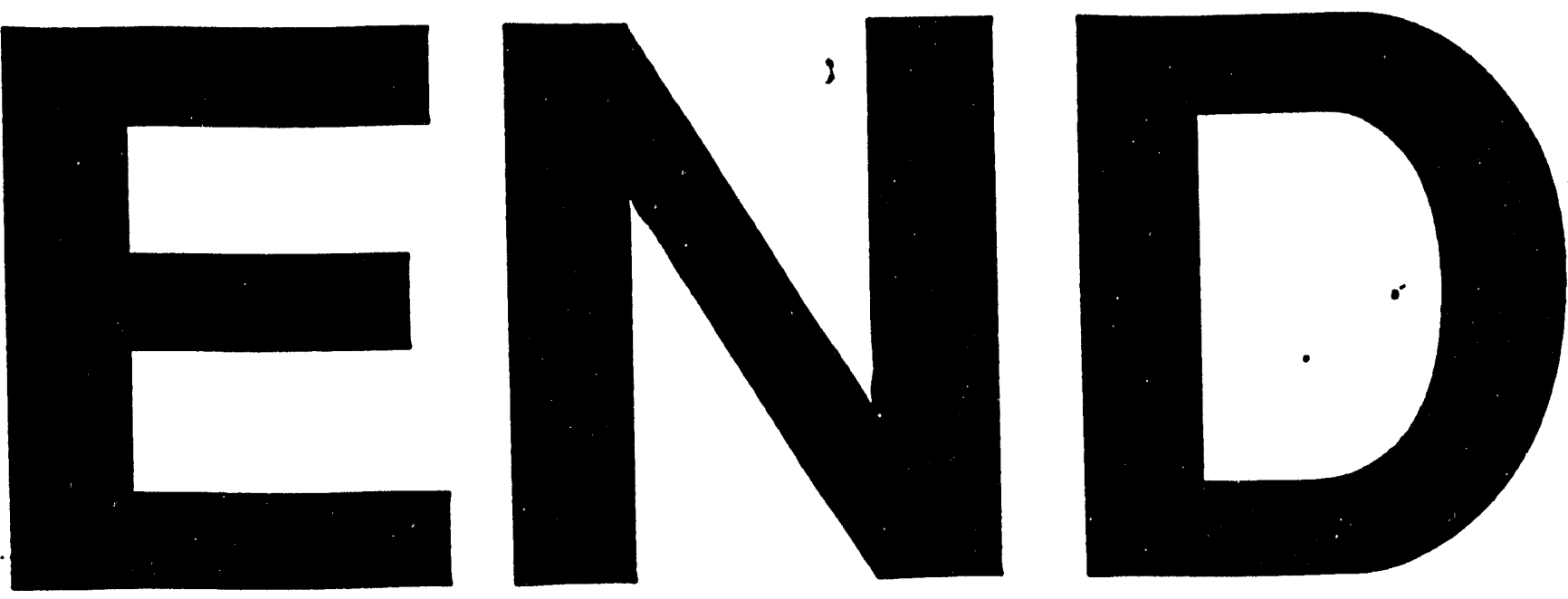
mom 